\title{
A Compact Circular Polarized Antenna for Deep Space Applications
}

\author{
M. Kirnamai, M. Sekhar
}

\begin{abstract}
A compact Circular polarized rectangular patch antenna has been designed for the Deep Space applications in the $\mathrm{Ku}$ band with an operating frequency of $13.15 \mathrm{GHz}$. The patch has been truncated at the diagonal corners and two $L$ shaped slots are been etched in the patch to get the circular polarization. A $50 \Omega$ coaxial cable has been used to excite the antenna. The overall dimension of the antenna is $13 \mathrm{~mm} \times 13 \mathrm{~mm} \times 0.508 \mathrm{~mm}$ which is $0.65 \lambda \times 0.65 \lambda$ making the proposed antenna a compact one. The Proposed antenna is having an impedance bandwidth of $25 \mathrm{GHz}$ ranging from $13 \mathrm{GHz}$ to $13.25 \mathrm{GHz}$ with a gain of $7.82 \mathrm{~dB}$ at the operating frequency of $13.15 \mathrm{GHz}$. The axial ratio of the antenna at the operating frequency is $2.94 \mathrm{~dB}$ which indicate the circular polarization. Low cost FR4 material is been used as the laminate base for the antenna which will act as the dielectric material.
\end{abstract}

Keywords-Compact, Circular Polarization, Deep Space Applications.

\section{INTRODUCTION}

To meet the requirement of the modern communication systems the need for antennas with compact size and circular polarization is increasing day by day and it has become a challenge for the designers to achieve circular polarization in a compact antenna by maintaining the necessary impedance bandwidth and gain. For Deep Space application circular polarization is a very essential parameter. It will reduce the signal loss due to polarization mismatch in between the transmitter and receiver antennas. It is also useful in reducing propagation losses.

In [1] an antenna with circular polarization has been proposed where the patch is been truncated for getting circular polarization but the antenna is fed with a microstrip feed line which will have low radiation efficiency because of the surface waves produced by the feed line as both the feed line and the radiating patch are on the same surface. In [2] an antenna with circular polarization has been proposed where the authors used two feeds to develop orthogonal modes to achieve circular polarization. But this technique needs two transmitter circuits which will affect the cost of the system. In [3] an antenna with a diagonal slot at the center has been proposed to achieve circular polarization but by adding slot in the radiating patch the radiation pattern of the antenna is not uniform and the power radiated by the antenna is not uniform over the entire region.

In [4] the authors proposed a technique to enhance the bandwidth of the antenna by considering a air gap in between the ground plane and the substrate.
In [5] a technique has been proposed to increase the gain of the antenna by taking a group of antennas and joining them as an array such that the power from all the individual elements will combine and form a single beam. In [6] Stacked patch techniques has been proposed which can be used for both gain enhancement and bandwidth enhancement depending upon the dimensions of the parasitic patch element. But all these techniques have complexity in designing either in terms of designing or in terms of cost. So to overcome these limitations we proposed a technique of Dielectric Resonator Antenna(DRA) where we use Dielectric material as a radiator instead of conductor. In [7] an four element antenna array has been proposed where all the four elements are been excited with a single feed and because of this the control over the phase of the input signal is not possible which is very essential in the phased array antenna applications especially in RADRA's where we need to steer the beam in different directions. In [8] an four element antenna array has been proposed where a separate feeding structure has been used to excite the individual antenna elements. For this proximity feeding concepts are been used which will require two different substrates for the development of the antenna and is not encouraged as it will increase the cost of the antenna. In [9] an four element antenna array has been proposed to achieve high gain but to excite the antenna elements a complex directional coupler network has been used and this is bringing difficulty in antenna fabrication and also the feed network is generating some surface waves which will reduce the overall efficiency of the antenna

In this paper, we proposed is a compact circular polarized antenna for $13.15 \mathrm{GHz}$ using Coax feed and to achieve circular polarization the radiating patch has been truncated at the two diagonal corners.

\section{DESIGN OF PROPOSED ANTENNA}

Proposed is a compact circular polarized antenna FOR 13.15GHz. Low loss Rogers RT Duroid 5880 material has been used as the substrate material with a thickness of $0.508 \mathrm{~mm}$. Substrate is a square with a side dimension of $13 \mathrm{~mm}$. The radiating patch is a rectnagle and is truncated at the corners diagonally by a square with side dimension of $1 \mathrm{~mm}$ along with it two $\mathrm{L}$ shaped slots to get the circular polarization. The coax feed position is orthogonal to the diagonal truncations. 


\section{A Compact Circular Polarized Antenna for Deep Space Applications}

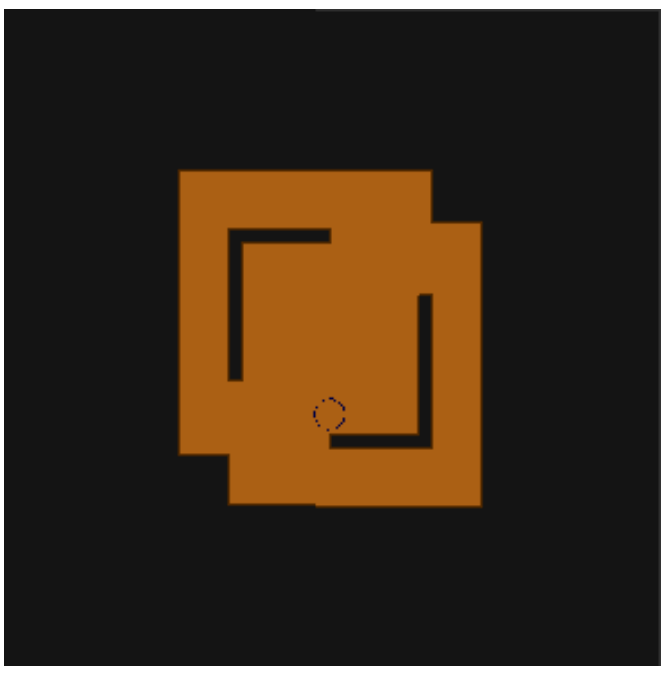

(a) Top View of proposed antenna

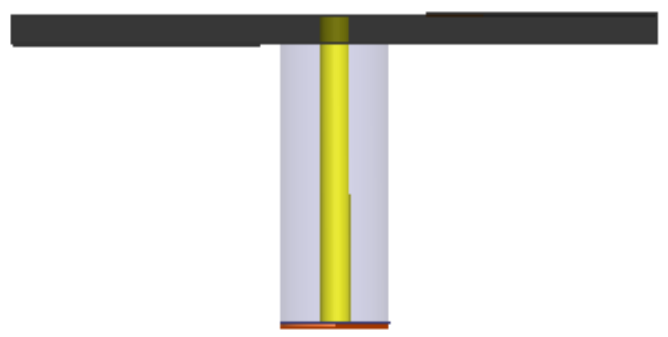

(b) Side View of proposed antenna

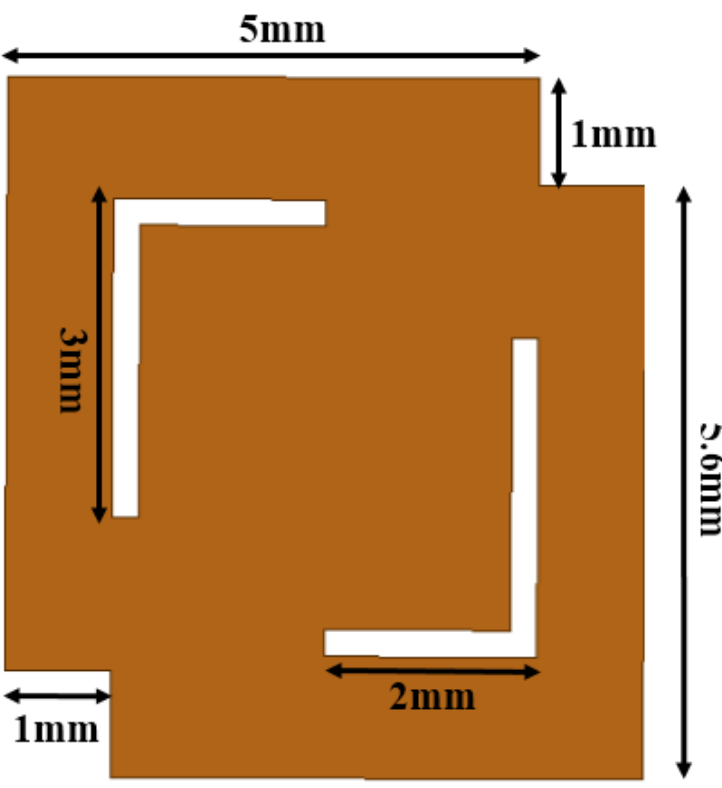

(c) Schematic Model of Patch Fig. 1. Antenna

\section{RESULTS AND DISCUSSION}

The performance of the antenna is measured by analyzing various parameters which includes impedance matching and radiation characteristics. Under impedance matching we will verify two parameters namely return loss also called as $S_{11}$ and Voltage standing wave ratio which is simply called as VSWR. [6-7]. Figure 3 and Figure 4 shows the impedance matching characteristics of the antenna.

Figure 3 shows the $S_{11}$ plot of the antenna from it we can know that the antenna is radiating in the frequency range of $13 \mathrm{GHz}$ to $13.25 \mathrm{GHz}$ with a operating centre frequency of
13.15GHz. It is observed that the return loss value is $-27 \mathrm{~dB}$ which indicated that there is a good impedance matching for the antenna with the input power source.

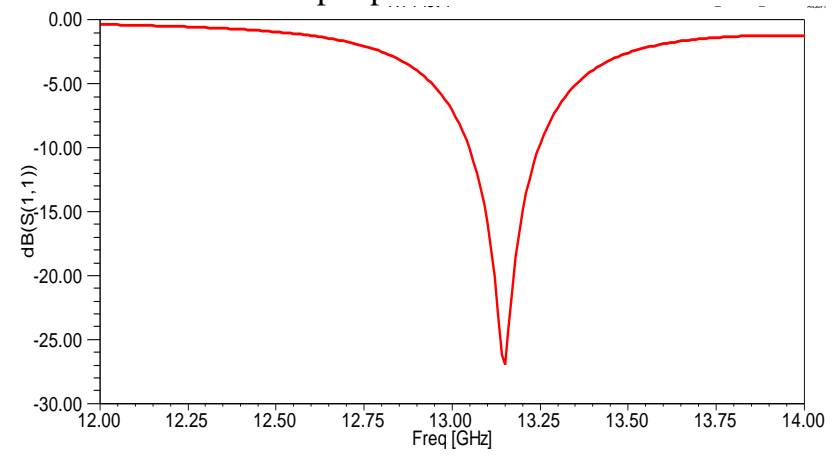

Fig. 3. Return loss

Figure 4 shows the VSWR plot of the antenna from it we can know that the antenna is radiating in the frequency range of $13 \mathrm{GHz}$ to $13.25 \mathrm{GHz}$ with a operating centre frequency of $13.15 \mathrm{GHz}$. It is observed that the VSWR value is $0.78 \mathrm{~dB}$ which indicated that there is a good impedance matching for the antenna with the input power source.

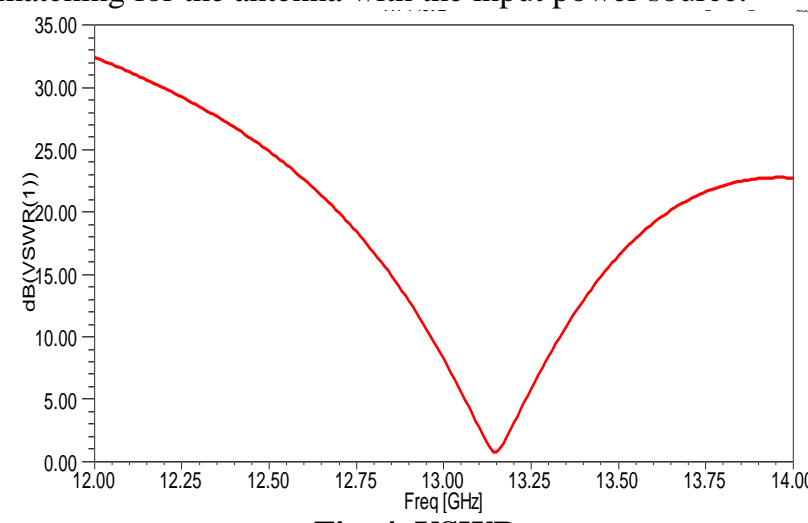

Fig. 4. VSWR

Figure 5, 6 and 7 below shows the 3D gain plot, 2D gain plot and the Directivity plot of the antenna at $13.15 \mathrm{GHz}$, The gain value of the antenna is $7.82 \mathrm{~dB}$ and the directivity of the antenna is $7.89 \mathrm{~dB}$. Figure 6 represents the two dimensional gain plot of the antenna it shows a uniform distribution of the power in different theta angles without any nulls which is very essential for the fixed communication applications $[8,9]$. The gain and directivity are nearly equal which represents that the losses are very low.
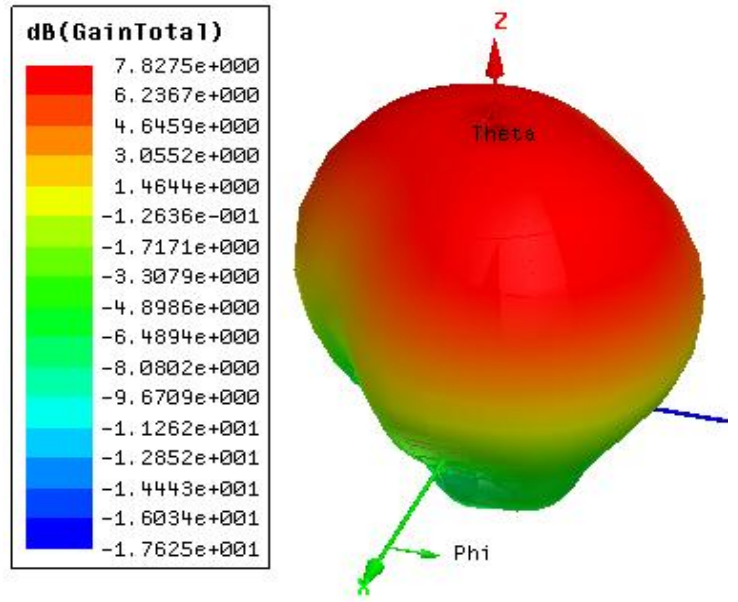

Fig.5. Gain at 13.15GHz 


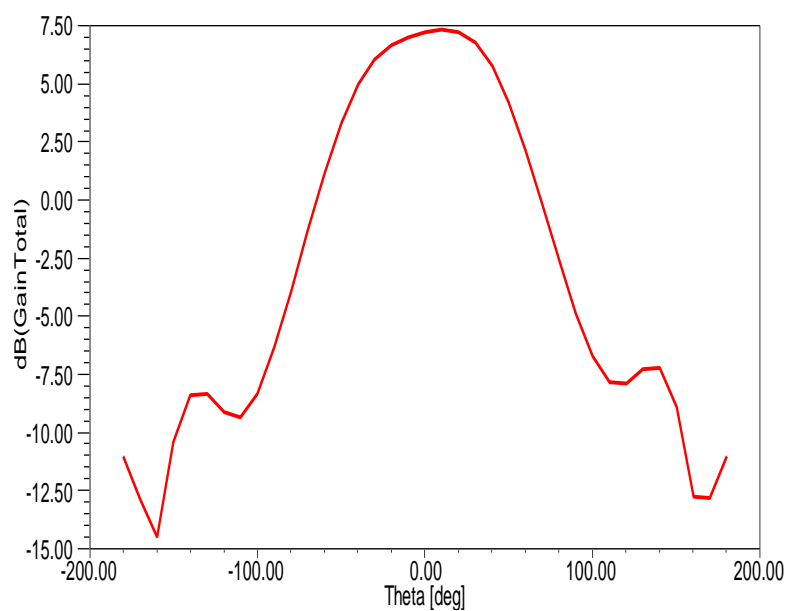

Fig. 6. Gain at $13.15 \mathrm{GHz}$
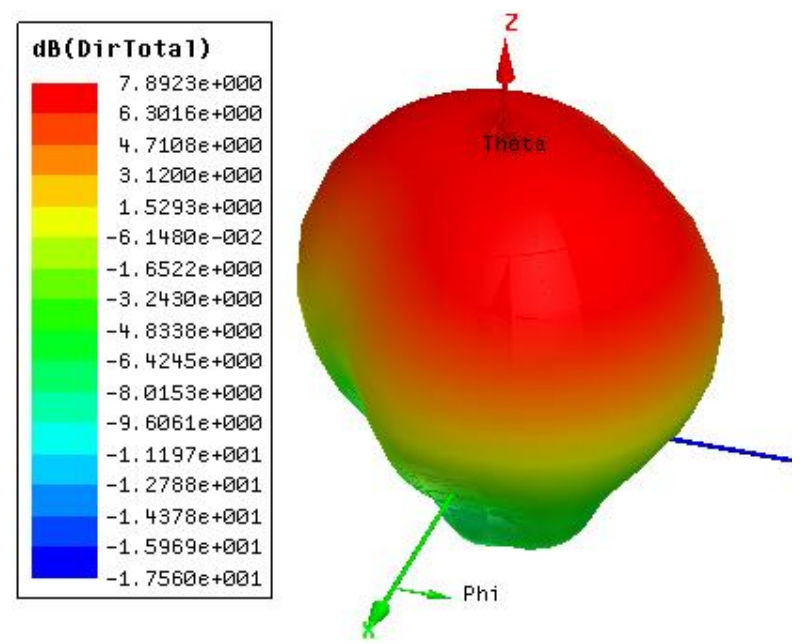

Fig.7. Directivity at $13.15 \mathrm{GHz}$

The radiation characteristics at the center frequency of 13.15GHz are shown below in Figures 7 and 8. To analyze this we need to check both the elevation plane and azimuthal. Both the patterns are having uniform distribution of the power in different theta angles without any nulls which is very essential for the fixed communication applications for proper planning of the antenna coverage area.

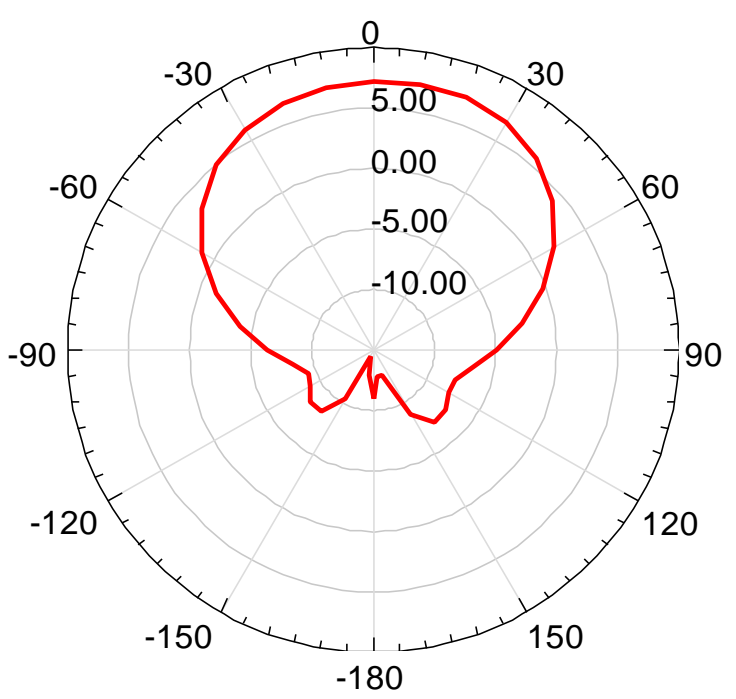

Fig. 10. Elevation Plane Patterns

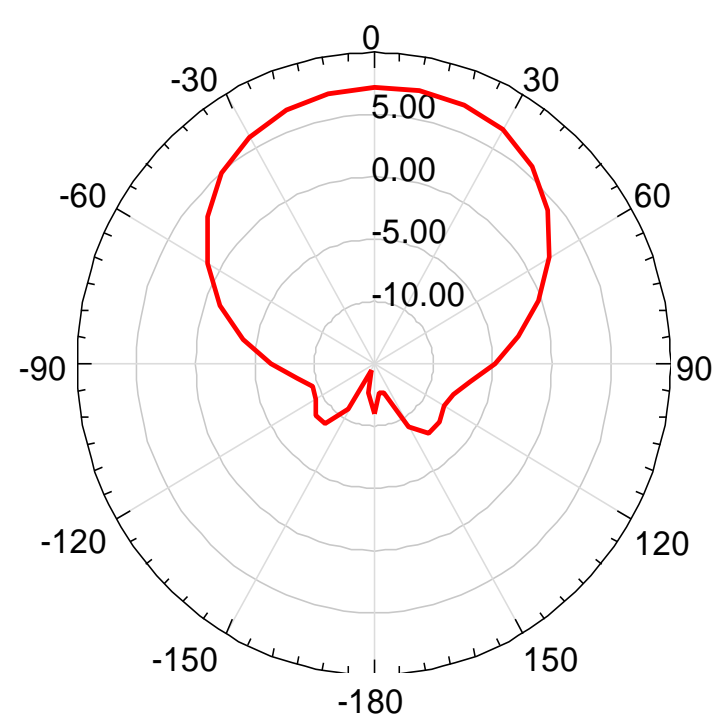

Fig. 11. Azimuthal Plane Patterns

Figure 10 below shows the Axial ratio pattern at $13.15 \mathrm{GHz}$. The axial ratio value is $2.94 \mathrm{~dB}$ at $0^{0}$ which conforms circular polarization.

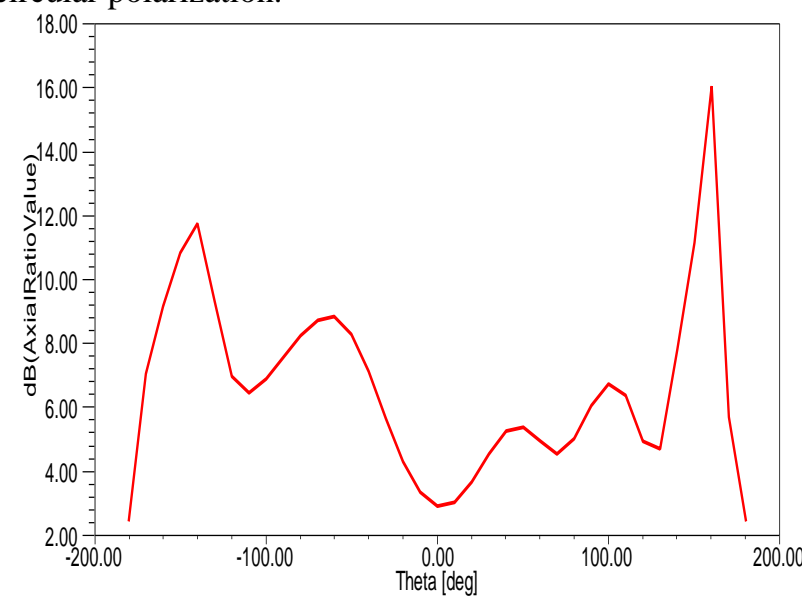

Fig. 10. Axial Ration at 13.15GHz

The radiation of the antenna depends on the current fields as presented in figure 11 below. We can observe that the intensity of the current field is minimum at the center of the patch and maximum at the edges. Figure 12 above shows the smith chart plot of the antenna.
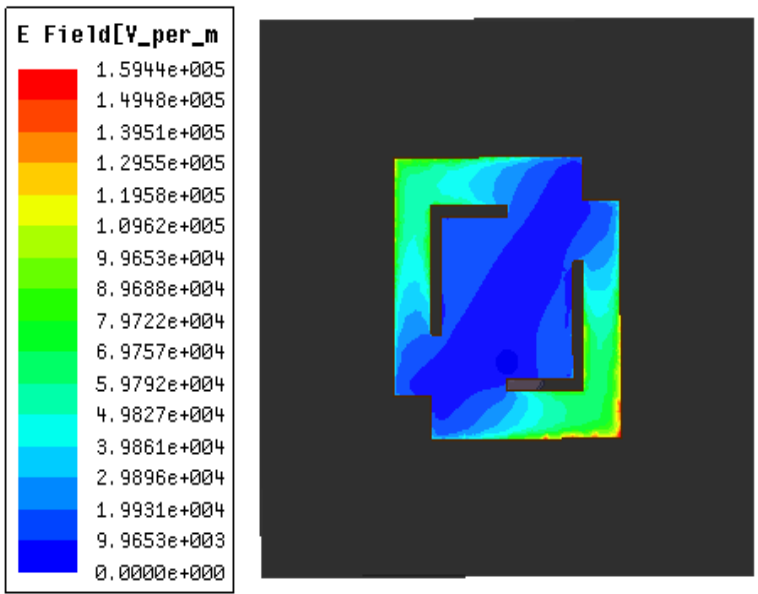

Fig. 11. Current distribution of the patch

Published By: 


\section{A Compact Circular Polarized Antenna for Deep Space Applications}

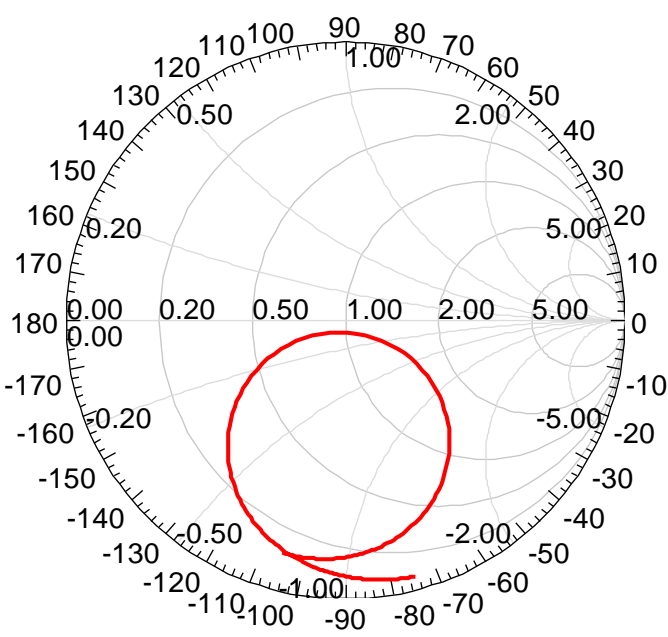

Fig. 12. Smith Chart

\section{CONCLUSION}

A compact Circular polarized antenna has been designed for Deep Space applications in the $\mathrm{Ku}$ band with an operating frequency of $13.15 \mathrm{GHz}$. The patch has been truncated at the diagonal corners and two $\mathrm{L}$ shaped slots are been etched in the patch to get the circular polarization. Rogers RT Duroid 5880 is taken as substrate material which is having a thickness of $0.508 \mathrm{~mm}$ and a $50 \Omega$ coaxial cable has been used to excite the antenna. The overall dimension of the antenna is $13 \mathrm{~mm} \times 13 \mathrm{~mm} \times 0.508 \mathrm{~mm}$ which is $0.65 \lambda \times 0.65 \lambda$ making the proposed antenna a compact one. The Proposed antenna is having an impedance bandwidth of $25 \mathrm{GHz}$ ranging from $13 \mathrm{GHz}$ to $13.25 \mathrm{GHz}$ with a gain of $7.82 \mathrm{~dB}$ at the operating frequency of $13.15 \mathrm{GHz}$. The axial ratio of the antenna at the operating frequency is $2.94 \mathrm{~dB}$ which indicate the circular polarization.

\section{REFERENCES}

1. E. Kusuma Kumari, A.N.V.Ravi Kumar. (2017). Wideband HighGain Circularly Polarized Planar Antenna Array for L Band Radar. IEEE International Conference on Computational Intelligence And Computing Research, Tamilnadu College of Engineering. Tamil Nadu.

2. E. Kusuma Kumari, A.N.V.Ravi Kumar. (2017). Development of an L Band Beam Steering Cuboid Antenna Array. IEEE International Conference on Computational Intelligence And Computing Research, Tamilnadu College of Engineering. Tamil Nadu.

3. Sunkaraboina Sreenu, Vadde Seetharama Rao. (2017). Stacked Microstrip Antenna For Global Positioning System. IEEE International Conference on Computational Intelligence And Computing Research, Tamilnadu College of Engineering. Tamil Nadu.

4. Rao N.A, Kanapala S. (2018). Wideband Circular Polarized Binomial Antenna Array for L-Band Radar. Panda G., Satapathy S., Biswal B., Bansal R. (eds) Microelectronics, Electromagnetics and Telecommunications. Lecture Notes in Electrical Engineering, vol 521. Springer, Singapore

5. Kanapala S, Rao N.A. (2018). Beam Steering Cuboid Antenna Array for L Band RADAR. Panda G., Satapathy S., Biswal B., Bansal R. (eds) Microelectronics, Electromagnetics and Telecommunications. Lecture Notes in Electrical Engineering, vol 521. Springer, Singapore.

6. Sunkaraboina Sreenu, P. Gnanasivam, M. Sekhar (2018). Circular polarised Antenna Array for C Band Applications. Journal of Advanced Research in Dynamical \& Control Systems, Vol. 10, 14 Special Issue.

7. K. Ashwini, M. Sekhar, Sunkaraboina Sreenu. (2018). Mutual Coupling Reduction Using Meander Square EBG Structures for CBand Radars. Journal of Advanced Research in Dynamical \& Control Systems, Vol. 10, 12-Special Issue.

8. Sekhar M, S Naga Kishore B, Siddaiah P. (2014). Triple Frequency Circular Patch Antenna. IEEE International Conference on
Computational Intelligence And Computing Research, Park College Of Engineering And Tekhnology. Tamil Nadu.

9. J Lavanya, S Nagakishore Bhavanam, Vasujadevi Midasala "Design of Spiral Antenna for Multiband Applications" International Journal of Innovative Technology and Exploring Engineering, Volume-8 Issue-5 March, 2019. 